\title{
Relação peso/largura da carapaça e fator de condição em Dilocarcinus pagei Stimpson (Crustacea, Trichodactylidae), em São José do Rio Preto, São Paulo, Brasil
}

\author{
Marcelo A. A. Pinheiro ${ }^{1} \&$ Fabiano G. Taddei ${ }^{2}$ \\ ${ }^{1}$ Grupo de Pesquisa em Biologia de Crustáceos, Universidade Estadual Paulista. Praça Infante Dom Henrique, \\ 11330-900 São Vicente, São Paulo, Brasil. E-mail: pinheiro@csv.unesp.br \\ ${ }^{2}$ Centro Universitário de Rio Preto. Rua Yvette Gabriel Atique 45, 15025-400 São José do Rio Preto, São Paulo, Brasil. \\ E-mail: fgtaddei@unirpnet.com.br
}

\begin{abstract}
Weight/carapace width relationship and condition factor in Dilocarcinus pagei Stimpson (Crustacea, Trichodactylidae) in São José do Rio Preto, São Paulo, Brazil. The biology of freshwater crabs has been little focused in the carcinology literature, mainly in relation to Dilocarcinus pagei Stimpson, 1861. This study aims to analyze weight/size relationship and temporal/seasonal variations of the condition factor, comparing with species biology. Individuals were monthly captured at São José of Rio Preto Dam's (São Paulo), during October 1994 to September 1996, and 962 specimens (534 males and 428 females) measured (CW = carapace width) and registered to weight (WW = total wet weight). Empirical points of WW/CW relationship were analyzed to each sex and submitted to regression analyses by power function $\left(y=a x^{b}\right)$, and represented by: $W_{\text {Males }}=0.0002 C W^{3.08}$ and $\mathrm{WW}_{\text {Females }}=0.0005 \mathrm{CW}^{2.82}$. Condition factor were monthly and seasonally calculated, with females' condition factor 2.5 times larger than of the males, due to the largest size/weight of the ovary. In general the summer was characterized by the smallest average of condition factor (reproductive period), contrasting with the largest verified in the winter (gonad reorganization). The results obtained are focused for the first time in the carcinological literature, being very important in population management and preservation of this species, that it comes being explored intensely and used as bait in the sporting fishing.
\end{abstract}

KEY WORDS. Freshwater crab, size.

RESUMO. A biologia de caranguejos de água doce tem sido pouco enfocada na literatura carcinológica, especialmente em relação à Dilocarcinus pagei Stimpson, 1861. Este estudo visa analisar a relação peso/tamanho e variações temporais/sazonais do fator de condição, comparando com a biologia da espécie. Os exemplares foram coletados mensalmente na Represa Municipal de São José do Rio Preto (São Paulo), durante um período bianual (outubro/1994 a setembro/1996). Foram obtidos 962 espécimes (534 machos e 428 fêmeas), que foram mensurados ( $L C=$ largura do cefalotórax) e pesados ( $\mathrm{PE}=$ peso úmido total). Os pontos empíricos da relação PE/LC foram analisados para cada sexo e submetidos à análise de regressão pela função potência $\left(y=a x^{b}\right)$ e representadas por: $\mathrm{PE}_{\text {Machos }}=0,0002 \mathrm{LC}^{3,08}$ e $\mathrm{PE}_{\text {Fêmeas }}=0,0005 \mathrm{LC}^{2,82}$. O fator de condição foi calculado mensal e sazonalmente, sendo o das fêmeas cerca de 2,5 maior que o dos machos, possivelmente devido ao maior tamanho/peso das gônadas. De modo geral o verão foi caracterizado pela menor média de fator de condição (época reprodutiva), contrastando com a maior verificada no inverno (reorganização gonadal). Os resultados obtidos são enfocados pela primeira vez na literatura carcinológica, sendo de grande valia no manejo populacional e preservação desta espécie, que vem sendo intensamente explorada e usada como isca na pesca esportiva. PALAVRAS CHAVE. Caranguejo de água doce, tamanho.

A relação peso/largura da carapaça tem sido empregada na interconversão entre variáveis, estimativa da variação do peso para determinado tamanho corpóreo e na detecção de alterações morfológicas ontogenéticas relacionadas ao início da maturação de crustáceos (Pinheiro \& Fransozo 1993). Nestes estudos, o modelo matemático comumente ajustado aos pontos empíricos da relação do peso úmido total pela largura cefalotorácica $(\mathrm{PE} / \mathrm{LC})$ é a função potência $(\mathrm{y}=\mathrm{ax}$ ) , o mesmo empregado nos estudos de crescimento relativo. No entanto, neste caso a constante "a" indica o grau de engorda (ou fator de condição), evidenciando variações do peso nas diferentes fases de crescimento, no início da maturidade sexual, entre se- 
xos e mesmo entre populações de diferentes localidades (SANTos 1978). A constante "b", por sua vez, reflete o tipo de crescimento em peso da espécie, podendo ser isométrico $(b=3)$, alométrico negativo $(\mathrm{b}<3)$ e alométrico positivo $(\mathrm{b}>3)$.

A análise da relação do peso em função do tamanho fornece informações essenciais para projeções de biomassa em aqüicultura, além de favorecer a exploração e manejo de espécies com importância pesqueira (SANTOS 1978). O fator de condição isométrico é determinado pela razão do peso úmido total do indivíduo pelo cubo de seu tamanho corpóreo, variando em função do teor de gordura, com o desenvolvimento gonadal e indicando o grau de adequação da espécie ao meio ambiente (le Cren 1951). No entanto, a maioria das espécies segue um padrão alométrico de crescimento em peso, requerendo que o tamanho seja elevado a constante "b" obtida na análise da relação PE/LC de cada sexo. Dentre os braquiúros já estudados, destacam-se os trabalhos de Costa et al. (1980), que avaliaram relações biométricas do peso/largura da carapaça para o siri Callinectes bocourti A. Milne-Edwards, 1879; Mori \& Zunino (1987) que analisaram aspectos da biologia de Liocarcinus depurator (Linnaeus, 1758); Branco \& Thives (1991) que determinaram o fator de condição para C. danae Smith, 1869; e Branco et al. (1992) que indicaram o período de desova de Callinectes danae através da interação do fator de condição com o índice gonadossomático. Outros autores como Pinheiro \& Fransozo (1993), Mantelatto \& Fransozo (1993) e Santos et al. (1995), descreveram a relação peso/largura da carapaça para Arenaeus cribrarius (Lamarck, 1818), Hepatus pudibundus (Herbst, 1785) e Portunus spinimanus Latreille, 1819, respectivamente. É importante destacar a originalidade deste artigo no que se refere a abordagem do assunto, tanto em relação a espécie em questão, como também para a Família Trichodactylidae H. Milne-Edwards, 1853 à qual pertence.

O objetivo do presente estudo é analisar a relação PE/LC para cada sexo de $D$. pagei, avaliar a dinâmica mensal e sazonal do fator de condição e associar os resultados obtidos com informações disponíveis sobre a biologia da espécie.

\section{MATERIAL \& MÉTODOS}

Os exemplares de $D$. pagei foram coletados mensalmente na Represa Municipal de São José do Rio Preto (São Paulo), com peneiras, puçás ou manualmente, durante um período de dois anos (outubro/1994 a setembro/1996), em associação com a vegetação marginal, com os aguapés Eichhornia azurea (Schwartz) Kunth, 1843 e $P$. crassipes (Martius) Solms, 1883 ou em galerias escavadas nos barrancos da represa.

Após a captura, os espécimes foram acondicionados em sacos plásticos etiquetados e transportados até o laboratório, onde foram mantidos sob congelamento até o momento das análises. Nesta ocasião, o material foi descongelado à temperatura ambiente e os indivíduos sexados e etiquetados. Embora D. pagei apresente dimorfismo abdominal entre os sexos (Melo 2003), a diferenciação entre jovens e adultos é impossível.
Cada exemplar teve sua largura cefalotorácica (LC) mensurada com um paquímetro $(0,05 \mathrm{~mm})$ e o peso úmido total (PE), registrado numa balança digital $(0,001 \mathrm{~g})$. Os espécimes que apresentavam injúrias morfológicas ou falta de pereiópodos foram descartados das análises.

Os pontos empíricos da relação PE/LC de cada sexo foram submetidos à análise de regressão, com ajuste pela função potência $(\mathrm{PE}=\mathrm{aLC})$, onde PE é a variável dependente, $\mathrm{LC}$ a independente, "a" é o grau de engorda ou fator de condição, e "b" uma constante associada ao tipo de crescimento em peso dos animais (HARTNOLl 1982).

Os dados obtidos durante o período de estudos para cada sexo, nas estações climáticas, permitiram a determinação das respectivas equações de PE/LC. Após a transformação logarítmica das variáveis, os valores dos coeficientes lineares (a) e angulares (b) foram confrontados por sexo, entre as estações, para verificar a existência de diferenças estatísticas pelo teste $t$ estudentizado (z), conforme SANTOS (1978). As médias sazonais do fator de condição também foram confrontadas pelo teste $t$, sob um nível de significância de 5\%.

\section{RESULTADOS}

Nas análises da relação PE/LC foram utilizados 863 indivíduos (456 machos e 407 fêmeas), com a média de tamanho e peso dos machos sendo significativamente maior que a das fêmeas $(\mathrm{p}<0,05)$ (Tab. I).

As variáveis PE e LC mostraram correlação positiva, com a tendência de seus pontos apresentando ajuste expressivo em todas as análises realizadas $\left(\mathrm{R}^{2} \geq 0,88\right)$ (Fig. 1). Os machos tiveram a relação $\mathrm{PE} / \mathrm{LC}$ expressa pela equação $\mathrm{PE}=0,0002 \mathrm{LC}^{3, \mathrm{o}} \mathrm{s}$ $\left(\mathrm{R}^{2}=0,96 ; \mathrm{N}=456\right)$, apresentando um crescimento em peso do tipo isométrico $(b=3,08)$, enquanto a equação das fêmeas foi $\mathrm{PE}=0,0005 \mathrm{LC}^{2,82}\left(\mathrm{R}^{2}=0,92 ; \mathrm{N}=407\right)$, sendo caracterizadas por um crescimento alométrico negativo $(\mathrm{b}=2,83)$.

O fator de condição das fêmeas foi cerca de 2,5 vezes superior ao dos machos, com a dinâmica das médias mensais evidenciando um padrão que se repetiu nos dois anos consecutivos. $\mathrm{Na}$ figura 2 verifica-se uma redução dos valores médios do fator de condição para ambos os sexos em dezembro (primeiro ano) e janeiro/fevereiro (segundo ano), enquanto em agosto ocorreram valores antagônicos, com elevação para os machos e redução para as fêmeas, particularmente no segundo ano de estudos.

A análise sazonal do fator de condição dos machos revelou uma maior média para o inverno, contrastando significativamente com a menor média que caracterizou o verão (Fig. 3), as quais não diferiram daquelas calculadas para a primavera e outono, similares entre si $(\mathrm{p}<0,05)$. Para as fêmeas as maiores médias foram obtidas para o outono e inverno, que foram similares entre si, diferindo da menor média sazonal (verão); estes valores médios foram similares ao obtido na primavera $(\mathrm{p}<0,05)$ (Fig. 3).

Na tabela II são apresentadas as equações obtidas por sexo e estações do ano, bem como o resultado do testes comparati- 

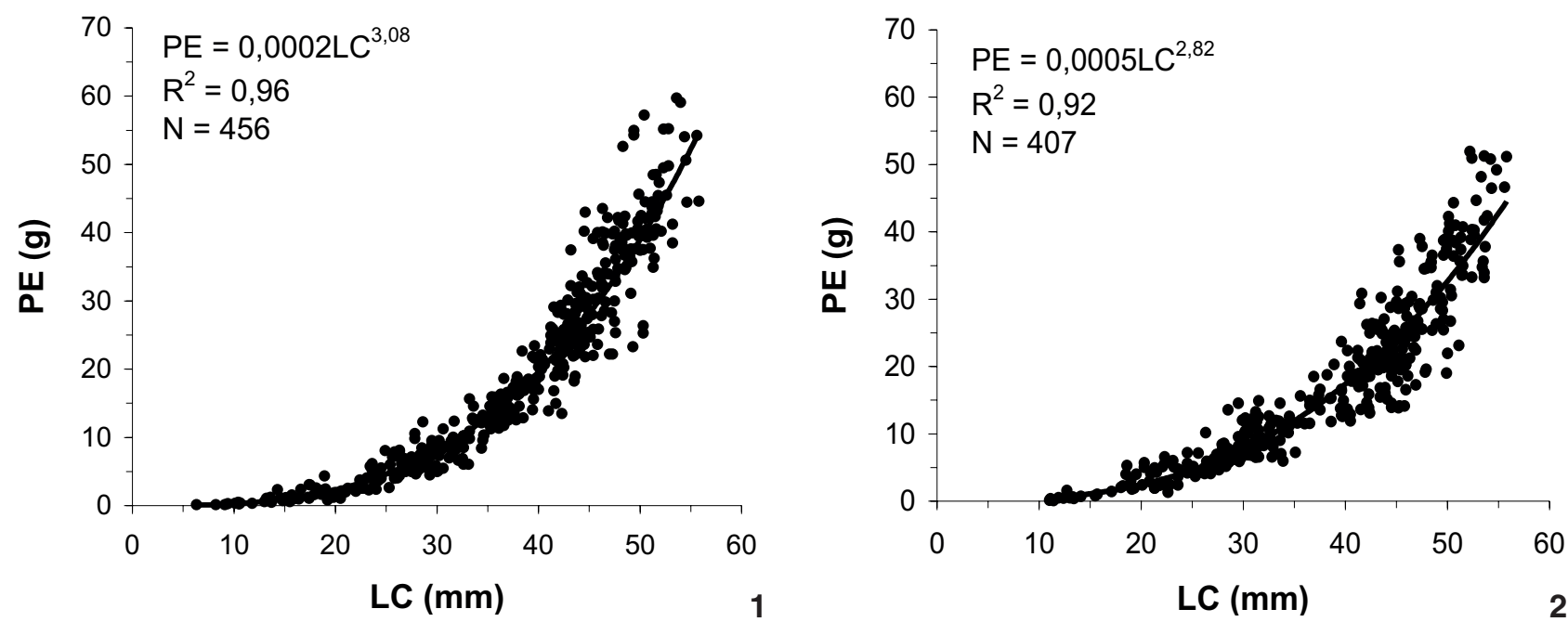

Figuras 1-2. Dilocarcinus pagei. Gráfico de dispersão de pontos da relação do peso úmido (PE) pela largura cefalotorácica (LC), para (1) machos e (2) fêmeas, capturados na Represa Municipal de São José do Rio Preto, São Paulo, no período de outubro/1994 a setembro/1996.

Tabela I. Dilocarcinus pagei. Sumário estatístico da largura da carapaça (LC) e peso úmido (PE), para ambos os sexos durante o período de outubro/1994 a setembro/1996.

\begin{tabular}{|c|c|c|c|c|}
\hline \multirow{2}{*}{ Parâmetros } & \multicolumn{2}{|c|}{$\mathrm{LC}(\mathrm{mm})$} & \multicolumn{2}{|c|}{ PE (g) } \\
\hline & Machos & Fêmeas & Machos & Fêmeas \\
\hline $\mathrm{N}$ & 534 & 428 & 456 & 407 \\
\hline Mínimo & 6,3 & 11,0 & 0,13 & 0,11 \\
\hline Máximo & 55,8 & 55,8 & 59,8 & 51,9 \\
\hline Média \pm Desvio Padrão & $38,2 \pm 10,6 a^{*}$ & $37,4 \pm 10,5 b$ & $20,5 \pm 14,3 a$ & $17,4 \pm 12,0 \mathrm{~b}$ \\
\hline
\end{tabular}

Médias de uma mesma variável biométrica seguidas por letras distintas mostraram contraste significativo $(p<0,05)$.

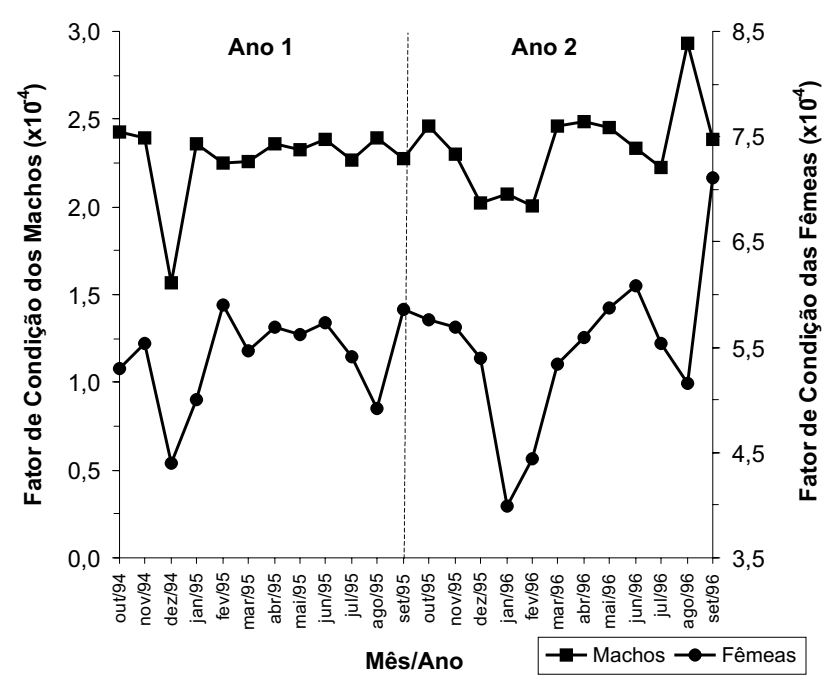

Figura 3. Dilocarcinus pagei. Gráfico de linha das médias mensais do fator de condição de cada sexo na Represa Municipal de São José do Rio Preto, São Paulo, durante o período de outubro/1994 a setembro/1996. vos entre suas constantes "a" e "b". Para os machos os índices de origem das equações não diferiram significativamente $\left(\mathrm{z}_{\text {calc }} \leq 1,48 ; \mathrm{p}>0,05\right)$, embora tenham ocorrido diferenças na inclinação das mesmas, sendo similares entre a primavera e inverno $\left(\mathrm{z}_{\text {calc }}=1,80 ; \mathrm{p}<0,05\right)$ e diferindo daquelas obtidas nas demais estações. Para as fêmeas os valores obtidos para "a" foram contrastantes $\left(z_{\text {calc }} \geq 9,83 ; p>0,05\right)$, enquanto os de " $b$ " apresentaram similaridade entre primavera/verão e verão/inverno $\left(3,19 \leq \mathrm{z}_{\text {calc }} \leq 5,82 ; \mathrm{p}<0,05\right)$.

\section{DISCUSSÃO}

O crescimento em peso é determinado ontogeneticamente, podendo variar entre os sexos, fases de maturação ou mesmo em populações de localidades distintas (HARTNOLL 1982). Segundo Pinheiro \& Fransozo (1993) a constante de crescimento em peso (b) é espécie-específica, ocorrendo alterações em função do mês, estação do ano ou geograficamente, em função da heterogeneidade ambiental.

A análise da relação PE/LC revelou que as constantes de crescimento em peso (b) determinadas para $D$. pagei estão den- 
Tabela II. Dilocarcinus pagei. Equações para a relação do peso úmido (PE) pela largura cefalotorácica (LC) para os machos e fêmeas, estimadas para cada estação do ano durante o período de outobro/1994 a setembro/1996.

\begin{tabular}{|c|c|c|c|c|c|c|c|}
\hline \multirow{3}{*}{$\begin{array}{c}\text { Estações } \\
\text { Primavera }\end{array}$} & \multicolumn{3}{|c|}{ Machos } & \multicolumn{4}{|c|}{ Fêmeas } \\
\hline & \multirow{2}{*}{$\frac{N}{92}$} & $\begin{array}{l}\text { Função Potência Linearizada } \\
\qquad(\ln y=\ln a+b \ln x)\end{array}$ & \multirow{2}{*}{$\begin{array}{c}\text { R2 } \\
0,89\end{array}$} & \multirow{2}{*}{$\frac{N}{98}$} & \multicolumn{2}{|c|}{$\begin{array}{l}\text { Função Potência Linearizada } \\
\qquad(\ln y=\ln a+b \ln x)\end{array}$} & \multirow{2}{*}{$\frac{\mathrm{R} 2}{0,90}$} \\
\hline & & $\ln \mathrm{PE}=-7,42+2,82 \ln \mathrm{LC} \quad \mathrm{A}(\mathrm{a})^{*}$ & & & $\ln P E=-6,38+2,49 \ln \mathrm{LC}$ & $B(b)$ & \\
\hline Verão & 133 & $\ln P E=-8,52+3,13 \ln \mathrm{LC} \quad \mathrm{A}(\mathrm{c})$ & 0,98 & 99 & In $P E=-8,52+3,12 \ln \mathrm{LC}$ & $A(d)$ & 0,96 \\
\hline Outono & 124 & $\ln P E=-8,52+3,10 \ln \mathrm{LC} A(b)$ & 0,95 & 104 & $\ln P E=-7,01+2,69 \ln \mathrm{LC}$ & $A B(c)$ & 0,89 \\
\hline Inverno & 107 & $\ln P E=-7,26+2,79 \ln \mathrm{LC} \quad \mathrm{A}(\mathrm{a})$ & 0,92 & 106 & $\ln P E=-5,95+2,39 \ln \mathrm{LC}$ & $\mathrm{B}(\mathrm{a})$ & 0,88 \\
\hline Total & 456 & $\ln P E=-8,52+3,08 \ln \mathrm{LC}$ & 0,96 & 407 & $\ln P E=-7,601+2,82 \ln \mathrm{LC}$ & & 0,92 \\
\hline
\end{tabular}

As equações sazonais obtidas para cada sexo seguidas de pelo menos uma mesma letra maiúscula não diferiram quanto ao coeficiente linear (a), o mesmo ocorrendo com as letras minúsculas no caso do coeficiente angular $(b)(p>0,05)$.

tro do limite estabelecido para outros organismos aquáticos $(2$ a 4, segundo Le Cren 1951 e Hartnoll 1982). Tais alterações dão indicativos de mudanças no peso dos organismos entre diferentes fases de desenvolvimento e no início da primeira maturação sexual (Mantelatto \& Fransozo 1992, Pinheiro \& Fransozo 1993).

A taxa de crescimento em peso dos machos de D. pagei foi superior à das fêmeas, similarmente ao obtido por SuMPTON et al. (1989) para Portunus sanguinolentus, Branco \& Thives (1991) para Callinectes danae e PinheIro \& Fransozo (1993) para Arenaeus cribrarius. No entanto, nestas espécies de Portunidae tal fato pode ser explicado pelo maior tamanho assintótico atingido pelos machos, o que não foi confirmado para D. pagei. Em função disso, o maior peso dos machos para um tamanho considerado pode ser resultante de dieta diferenciada, maior taxa de conversão alimentar ou mesmo pela presença da glândula andrógena nos machos, responsável pelo aumento em peso logo após a maturidade (BuIss 1968).

A menor taxa de crescimento em peso das fêmeas de $D$. pagei $(b=2,82)$, minimiza o valor do denominador da relação $\mathrm{PE} / \mathrm{LC}^{\mathrm{b}}$, gerando valores de fator de condição cerca de 2,5 vezes maiores dos que o dos machos, certamente em função do maior tamanho e peso dos ovários, que quando maturos superaram em quase três vezes o tamanho dos testículos em machos de porte similar.

Barbieri \& Verani (1987) mencionam que as oscilações temporais do fator de condição em peixes teleósteos trazem informações importantes sobre a reprodução, já que os maiores valores são associados à maturação das gônadas, enquanto os menores caracterizam sua reorganização logo após a desova. Como as fêmeas dos caranguejos estocam os espermatóforos em um par de espermatecas, no momento da cópula as gônadas dos machos devem estar maturas, o mesmo não sendo necessário para as fêmeas, que têm seus ovários em desenvolvimento nesta ocasião (Pinheiro \& Fransozo 2002). Tal fato pode explicar os valores antagônicos do fator de condição registrados em agosto, bem como sua inversão em setembro, quando deve ocorrer a cópula da espécie. A redução do fator de condição da espécie do final da primavera a meados do verão pode ser explicada pela liberação dos jovens pela fêmea, já que a espécie apresenta um desenvolvimento do tipo epimórfico (KAESTNER 1970), enquanto para os machos a hipótese mais plausível seria a troca de exosqueleto (época de muda).

O peso e volume dos órgãos das fêmeas podem variar em função do desenvolvimento ovariano, bem como ao decréscimo das reservas energéticas do hepatopâncreas e em função da extrusão dos ovos (HAEFNER \& SPAARGAREN 1993), esclarecendo porque se reduzem nos meses que antecedem a reprodução, quando grande parte da reserva energética é direcionada às gônadas. Os dados do presente estudo se coadunam com o desses autores, ocorrendo o mesmo com os peixes (Agostinho et al. 1990).

Apesar da reduzida variação das médias sazonais do fator de condição verificadas para cada sexo, foi possível determinar que a época reprodutiva de D. pagei ocorre na primavera/verão (menores valores), o que é corroborado pelo registro de fêmeas ovígeras ou portando jovens no abdome, que ocorrem somente nestes meses. Os dados obtidos confirmam as observações de Branco et al. (1992) sobre a coincidência dos meses de maior fotoperíodo/pluviosidade com os de menor fator de condição. Apesar disso, estes autores indicam tais períodos como de reprodução de uma espécie, fato válido para espécies de reprodução sazonal, como D. pagei, mas não para braquiúros com reprodução contínua.

A análise do fator de condição e as informações que dela advém sobre a época reprodutiva, bem estar e adaptação do animal ao ambiente, faz com que tais avaliações venham a contribuir para um futuro cultivo da espécie enfocada, visto que auxiliam a melhor compreensão dos processos biológicos e ecológicos que regem seu desenvolvimento. O IBAMA do Estado de Mato Grosso do Sul, tem se preocupado com a extração contínua deste recurso por catadores que capturam exemplares juvenis desta espécie nas lagoas marginais de rios do Pantanal, vendendo-os como isca viva na pesca do Pacú. Esta prática tem causado impacto às lagoas marginais, que têm sua vegetação e sedimento revolvido, além da própria população de $D$. pagei, que pode entrar em declínio em resposta a intensidade de captura. 


\section{AGRADECIMENTOS}

Ao Prof. Dr. Adilson Fransozo (Depto. de Zoologia, IB, UNESP Botucatu) pelo apoio e incentivo, ao Prof. Dr. Edmir Daniel Carvalho (Depto. Morfologia, IB, UNESP Botucatu) pelas proveitosas explicações sobre os testes de comparação das equações matemáticas, e ao MSc. Gustavo Y. Hattori por seu auxílio na análise de dados e pela amizade.

\section{REFERÊNCIAS BIBLIOGRÁFICAS}

Agostinho, A.A.; G. Barbieri; J.R. Verani \& N.S. Hanh. 1990. Variação do fator de condição e do índice hepatossomático e suas relações com o ciclo reprodutivo em Rhinelepis aspera (Agassis, 1829) (Osteichthyes, Loricariidae) no Rio Paranapanema, Porucatu, PR. Ciência e Cultura, São Paulo, 42 (9): 711-714.

BARbieri; G. \& J.R. Verani. 1987. O fator de condição como indicador do período de desova em Hypostomus aff. plecostomus (Linnaeus, 1758) (Osteichthyes, Loricariidae), na Represa do Monjolinho (São Carlos, SP). Ciência e Cultura, São Paulo, 39 (7): 655-658.

BuIs, D.E. 1968. Transition From Water to Land in decapod Crustaceans. American Zoologist, Lawrence, 8: 355-392.

Branco, J.O. \& A. Thives. 1991. Relação peso/largura, fator de condição e tamanho da primeira maturação de Callinectes danae Smith, 1869 (Crustacea, Portunidae) no manguezal do Itacorubi Mangrove, SC, Brasil. Arquivos de Biologia e Tecnologia, Curitiba, 34 (3/4): 415-424.

Branco, J.O.; M.J. Lunardon; M.G. Avila \& C.F. Miguel. 1992. Interação entre fator de condição e índice gonadossomático como indicadores do período de desova em Callinectes danae Smith (Crustacea, Portunidae) da Lagoa da Conceição, Florianópolis, Santa Catarina, Brasil. Revista Brasileira de Zoologia, Curitiba, 9 (3/4): 175-180.

Costa, F.J.C.B.; I.V. Nascimento \& M.F.P. SÁ. 1980. Estudo biométrico do siri gurjaú, Callinectes bocourti A. Milne Edwards, 1879, da Lagoa Manguába. Boletim do Núcleo de Estudos de Ciências do Mar, Maceió, 2: 5-11.

Haefner JR., P.A. \& D.H. SpaArgaren. 1993. Interactions of ovary and hepatopancreas during the reproductive cycle of Crangon crangon (L.) I. Weigth and volume relationships. Journal of Crustacean Biology, San Antonio, 13 (3): 523-531.

Hartnoll, R.G. 1982. Growth, p. 111-185. In: D.E. Bliss (Ed.).
The Biology of Crustacea. Embriology, Morphology and Genetics. New York, New York Academic Press, vol. 2, 383p.

KaEsTner, A. 1970. Invertebrate Zoology. New York, Crustacea Interscience Publishers, vol. 3, 523p.

LE CREN, E.D. 1951. The lenght-weight relationship and seasonal cycle in gonad weight and condition in the perch (Perca fluviatilis). Journal of Animal Ecology, Oxford, 20 (2): 201219.

Mantelatto, F.L.M. \& A. Fransozo. 1992. Crescimento relativo e dimorfismo sexual em Hepatus pudibundus (Herbst, 1785) (Decapoda, Brachyura) no litoral norte paulista. Arquivos de Biologia e Tecnologia, Curitiba, 35 (4): 719-724.

Mantelatto, F.L.M. \& A. Fransozo. 1993. Relação peso/largura da carapaça no caranguejo Hepatus pudibundus (Herbst, 1785) (Crustacea, Decapoda, Callapidae) of the Ubatuba region, SP, Brasil. Arquivos de Biologia e Tecnologia, Curitiba, 35 (4): 719-724.

Melo, G.A.S. 2003. Manual de Identificação dos Crustacea Decapoda de Água Doce do Brasil. São Paulo, Edições Loyola, 429p.

Mori, N. \& P. Zunino. 1987. Aspects of the biology of Liocarcinus depurator (L.) in the Ligurian Sea. Investigacion Pesquera, Barcelona, 51: 135-145.

Pinheiro, M.A.A. \& A. Fransozo. 1993. Análise da relação biométrica do peso úmido pela largura da carapaça para o siri Arenaeus cribrarius (Lamarck, 1818) (Crustacea, Brachyura, Portunidae). Arquivos de Biologia e Tecnologia, Curitiba, 36 (2): 331-341.

Pinheiro, M.A.A. \& A. Fransozo. 2002. Reproduction of the speckled swimming crab Arenaeus cribrarius (Brachyura: Portunidae) on the Brazilian coast near $23^{\circ} 30^{\prime}$ S. Journal of Crustacean Biology, San Antonio, 22 (2): 416-428.

SAntos, E.P. 1978. Dinâmica de Populações Aplicada à Pesca e Piscicultura. São Paulo, HUCITEC/EDUSP, 129p.

Santos, S.; M.L. Negreiros-Fransozo \& C.R. Padovani. 1995. Relação do peso do corpo em função da largura da carapaça do siri candeias Portunus spinimanus Latreille, 1819 (Decapoda, Portunidae). Arquivos de Biologia e Tecnologia, Curitiba, 38 (3): 715-724.

Sumpton, W.D.; G.S. Smith \& M.A. Potter. 1989. Notes on the biology of the portunid crab, Portunus sanguinolentus (Herbst), in subtropical queensland waters. Australian Journal of Marine and Freshwater Research, East Melbourne, 40: 711-717.

Recebido em 31.VIII.2004; aceito em 13.VI.2005. 